\title{
A Biomechatronic Device Actuated by Pneumatic Artificial Muscles for the Automatic Evaluation of Nociceptive Thresholds in Rheumatic Patients
}

\author{
Luigi Randazzini ${ }^{1}{ }^{(D}$, Alessia Capace ${ }^{1}\left(\mathbb{D}\right.$, Carlo Cosentino ${ }^{1, *(\mathbb{D})}$, Rosa Daniela Grembiale ${ }^{2}(\mathbb{D}$, \\ Francesco Amato ${ }^{3}{ }^{(1)}$ and Alessio Merola ${ }^{1}$ (1) \\ 1 Biomechatronics Laboratory, Department of Experimental and Clinical Medicine, Università degli Studi \\ Magna Græcia di Catanzaro, Campus Universitario “S. Venuta”, 88100 Catanzaro, Italy; \\ randazzini@unicz.it (L.R.); alessia.capace@unicz.it (A.C.); merola@unicz.it (A.M.) \\ 2 Rheumatology Research Unit, Department of Health Sciences, Università degli Studi Magna Græcia di \\ Catanzaro, Campus Universitario "S. Venuta", 88100 Catanzaro, Italy; rdgrembiale@unicz.it \\ 3 Dipartimento di Ingegneria Elettrica e delle Tecnologie dell'Informazione, Università degli Studi di Napoli \\ Federico II, Via Claudio 21, 80125 Napoli, Italy; francesco.amato@unina.it \\ * Correspondence: carlo.cosentino@unicz.it; Tel.: +39-0961-369-4051
}

Received: 23 July 2020; Accepted: 26 August 2020; Published: 28 August 2020

check for updates

\begin{abstract}
In the current clinical practice, the diagnosis of Rheumatoid Arthritis (RA) draws on the qualitative evaluation of pain sensitivity thresholds which is affected by several source of uncertainties, due to an operator-dependent diagnostic protocol. Taking our cue from the diagnostic shortcomings, we have explored the possibility of automating the measurement of mechanical nociceptive thresholds through the adoption of soft pneumatic actuators controlled by an embedded control unit. In this work, we want to show that a purpose-made biomechatronic device actuated by soft and pneumatic actuators is potentially a boon both for rheumatologists and biomedical researchers involved in nociception and physicophysical studies. In the full breadth and scope of the objective diagnosis of RA, the first prototype of a novel biomechatronic device for quantitative and automatic measurement of mechanical nociceptive thresholds has been designed and tested through nociception experiments on 10 subjects. The experimental results show that the designed device can reliably generate the controllable and repeatable nociceptive stimuli needed for the objective diagnosis of RA.
\end{abstract}

Keywords: pneumatic artificial muscle; soft robotics; haptic devices; automated diagnosis; rheumatic diseases

\section{Introduction}

In the last few years, flexible actuators and soft robots have gained increasingly importance thanks to their inherent compliance and adaptability of the control performance useful in many biorobotic applications. For instance, within the fields of robotic rehabilitation and biorobotic prosthetics, there is a growing need of devices which interact safely with humans.

Unlike standard electric and hydraulic drives, soft actuators exhibit a compliant behavior guaranteeing intrinsically safe interaction. The design of biorobotic and soft actuators, which is often adaptable to the structure of the joints of human limbs and articulations, is inspired by the biomimetic replication of the biomechanics of the human skeletal structure actuated by multiple muscles. Moreover, in the event of failure of the actuator, the risk of injury to the patient is lower when soft (and pneumatic) actuators are adopted, as an alternative to electric/hydraulic actuators, for the control of wearable biorobots and robotic prostheses. These motivations foster the development of soft 
actuation technologies for all the diagnostic and therapeutic applications which require the control of the interaction between the patient and the compliant mechanisms, e.g., of flexible endoscopic tools, exoskeletons, wearable, and haptic devices.

The central challenge on the design of soft actuators is to develop some actuation solutions combining good dynamical performance and reliability. Nowadays, soft robotics actuation technologies make use of pneumatic chambers, electroactive polymers, electrorheological and magnetorheological materials, shape-memory alloys, and, more recently, microfabricated biomimetic actuators.

The review papers in [1-3] examine, in depth, the most recent advancements in the actuation of soft robots for biomedical applications. Among several actuation technologies, Flexible Fluidic Actuators (FFAs) are firstly investigated. FFAs, which consist of a flexible structure actuated by a fluid, are used in many biorobotic applications, such as rehabilitation and assistance, surgery, drug delivery, and wearable haptic devices.

Pneumatic Artificial Muscles (PAMs) are soft and biomimetic actuators, as their behavior replicates that of the biological muscles which generate, only under contraction, the actuation force. The elastic chamber of a PAM is filled by pressurized air to obtain a contraction force.

PAMs, which are a subgroup of FFAs, guarantee their versatile applicability as light, compliant, and inherently safe actuators for wearable and rehabilitation exoskeletons (see, e.g., in [4-6]). Both fabrication technologies and model-based control methodologies are now mature for the development of biorobotic devices actuated by PAMs. More recently, the results in $[7,8]$ show how the modeling of the mechanical response of PAMs may improve the control performance of servomechanisms actuated by fluidic muscles.

Pneumatic actuation technologies, ranging from pneumatic balloon actuators to PAMs, are implemented into the most efficient examples of design of wearable haptic devices. In these example, some soft and inflatable structures, which are in contact with the dorsal and palmar surfaces of the hand in order to generate kinesthetic and tactile stimuli, are generally shaped as gloves or exoskeleton adaptable to the hand anatomy [9-15].

Soft robotics can beneficially be applied to the diagnosis of Rheumatoid Arthritis (RA), which is a chronic disease characterized by systemic inflammatory conditions affecting synovial joints, mostly at the ends of the upper extremities of the human musculoskeletal system [16]. The disease progression can evolve from painful and swollen articulations to the impairment of the hands. RA, which mainly affects $30-50$ year-old females, has an estimated incidence of $\sim 1 \%$ with global geographical distribution [17]. The intense sensitization of the synovial joints results in the lower pain thresholds of rheumatic patients.

The standard diagnostic and therapeutic protocols, which are adopted in rheumatological practice, are based on the evaluation of the patient's sensitivity threshold in response to a mechanical nociceptive input. Therefore, the patient's pain threshold is one of the parameters involved into the estimation of the severity of RA [18]. The pain sensitivity threshold is estimated through the manipulation of the articular joints of the patient; an increasing compression is exerted by the rheumatologist on the synovial joints, e.g., of the patient's hand, in order to elicit a sensation of pain. The patient assigns, on a discrete scale within the interval [0-10], said Visual Analogic Scale (VAS), a score to the perceived pain. The standard diagnosis of RA is not robust since the measurement of the pain threshold in rheumatic patients is affected by some sources of uncertainty, due to the interindividual variability of the pain perception and mainly because of an operator-dependent protocol [19].

This paper presents and discusses the main functional features and the performance of an innovative biomechatronic device, which is designed to help the physician by providing a support for quantitative and standardized diagnosis of RA.

The goal of reducing the diagnosis uncertainty can be achieved through the device, mainly through the automation of the measurement of the nociceptive thresholds in rheumatic patients. This achievement has been reached by exploiting all the advantages deriving from the adoption of pneumatic actuation and soft robotics technologies. Indeed, some PAMs actuate the forces required for 
generating, in a controllable and measurable way, the mechanical nociceptive stimuli on the synovial joints of a subject.

The device includes a multichannel interface for nociceptive signal generation and digital data acquisition, other than a microcontroller-based control unit for the regulation of the force on the hand joints. Moreover, some embedded Bluetooth Low Energy (BLE) and Wi-Fi communication modules enable the microcontroller programming other than the telemonitoring of diagnostic sessions in a custom cloud service.

The device has been designed, built and tested at the Biomechatronics Laboratory of the University of Catanzaro "Magna Græcia", Catanzaro, Italy. The first prototype of the device is a tangible result of the cooperation between bioengineers and rheumatologists, starting from a critical review of the current limitations of standard diagnostic protocols.

The experimental validation of the effectiveness and repeatibility of a protocol, for the quantitative measurement of pain sensitivity through the biomechatronic device, has been accomplished.

The paper is organized as follows. Section 2 provides an overview of the current protocols for RA diagnosis. Section 3 presents the biomechatronic device in its main components and functions; Section 4 presents the experimental set-up. Section 5 summarizes and discusses the results of the nociception experiments devoted to the validation of the prototype and of the measurement protocol. Finally, some concluding remarks and plans for future work are given in Section 6.

\section{Evaluation of the Mechanical Nociceptive Threshold: State-of-the-Art in Rheumatology}

The inflammatory disorders affecting the synovial joints of rheumatic patients range from low effects to chronic musculoskeletal pain and sensitization in the peripheral and central pathways of the nervous system. The objective evaluation of severity and progression of RA highlights the importance of the standardization of the protocols for the measurement of the mechanical nociceptive thresholds in rheumatic patient. Moreover, the definition of clinical protocols based on the quantitative measurement of pain sensitivity thresholds has important implications both for the diagnostic accuracy and therapy planning.

During the standard tests on mechanical nociceptive sensitivity, the rheumatologist performs a manual (and not measurable) compression on the articular joints of the patient. The Disease Activity Score 28 (DAS 28) scale is recognized by the American College of Rheumatology [18] as one of the gold standards for the classification of RA severity. The diagnostic criteria of the RA severity, which is based on the DAS 28 scale, is summarized in Table 1. DAS 28 is an index mixing the VAS score, biomolecular information from the disease markers of erythrocyte sedimentation rate, reactive $C$ protein and, furthermore, the number of swollen and tender joints in correspondence of metacarpophalangeal joints, proximal interphalangeal articulations of the hands, shoulders, elbows, wrists, and knees (see Figure 1).

Table 1. DAS 28 scale: classification of RA severity.

\begin{tabular}{cc}
\hline Disease Severity & Index Range \\
\hline remission & DAS $\leq 2.6$ \\
low & $2.6<\mathrm{DAS} \leq 3.2$ \\
moderate & $3.2<\mathrm{DAS} \leq 5.1$ \\
high & $\mathrm{DAS}>5.1$ \\
\hline
\end{tabular}

DAS 28 index can be calculated as

$$
D A S 28=0.56 \sqrt{t 28}+0.28 \sqrt{s w 28}+0.70 \log E S R+0.014 G H,
$$


where $t 28$ and $s w 28$ indicate the number of tender and swollen joints, respectively; ESR is the erythrocyte sedimentation rate in $\mathrm{mm} /$ hours and $G H$ is the Global Health parameter representing the patient's self assessment of disease activity through VAS evaluation.

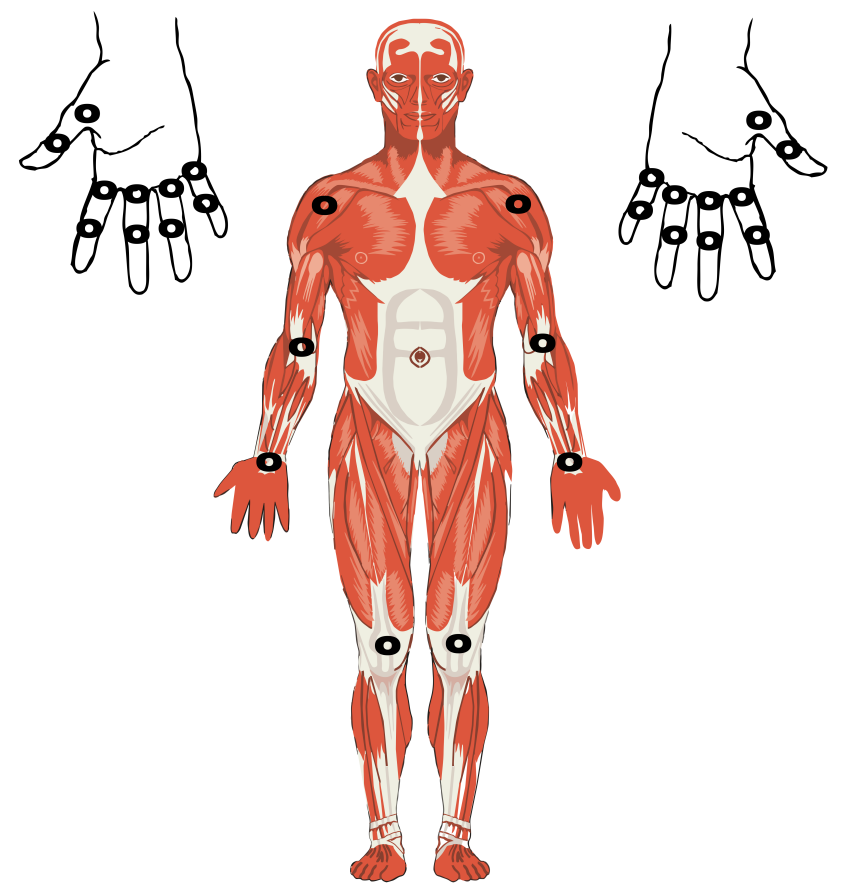

Figure 1. Measurement points in DAS 28 protocol.

Most of the acquisitions of pain sensitivity thresholds are taken in correspondence of the articulations of the hands of Figure 1, as the inflammatory response is most frequently localized at the finger joints.

\section{Biomechatronic Design of the Robotic Exoskeleton for Rheumatological Diagnosis}

\subsection{Soft Robotic Hardware}

The PAMs involved in the proposed biomechatronic solution have a relevant impact on the specific application of guided diagnosis of RA. Indeed, the inherent compliance of PAMs enables the safe control of the interaction between the device and the patient, in addition to some constraints on the upper bound of the actuation pressure which are implemented into the embedded controller of the device. Moreover, in the design of the mechanical hardware of the device, an efficient solution similar to grounded haptic platforms has been adopted, e.g., see in [20] Figure 1a, in which both the dimensions and the number of mechanical components superimposed on the patient's fingers are minimized. Therefore, the resulting prototype possesses marked characteristics of wearability, compactness, and lightness in order to reduce the discomfort to swollen and painful articulations.

The main parts of the device, which are illustrated in Figure 2, are the stimulation module at the patient's side and the control and actuation unit enclosing PAMs, electro-pneumatic component's and microcontrollers with BLE+Wi-Fi communication modules.

The device has some elastic rings placed on the articular joints of the hands of a rheumatic patient (see Figure 3). The rings surround the joint cavities of the patient's finger receiving a mechanical stimulus through the deformation of the same rings. The nociceptive stimulus is generated through a radial compression over the perimeter of the sinovial cavities of the patient's finger; this set-up replicates the manual compression actuated by the physician's fingers on the skin and soft tissues of the patient. 


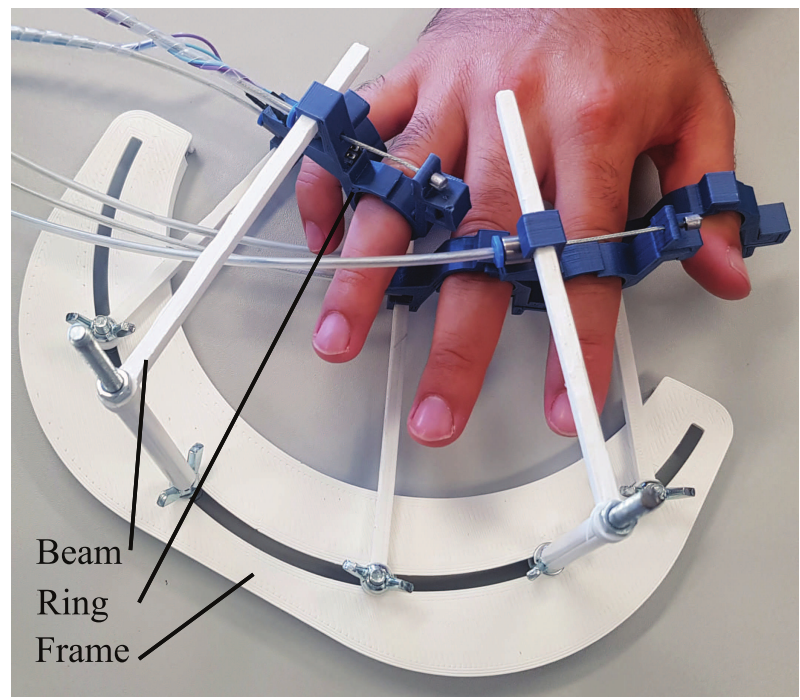

(a)

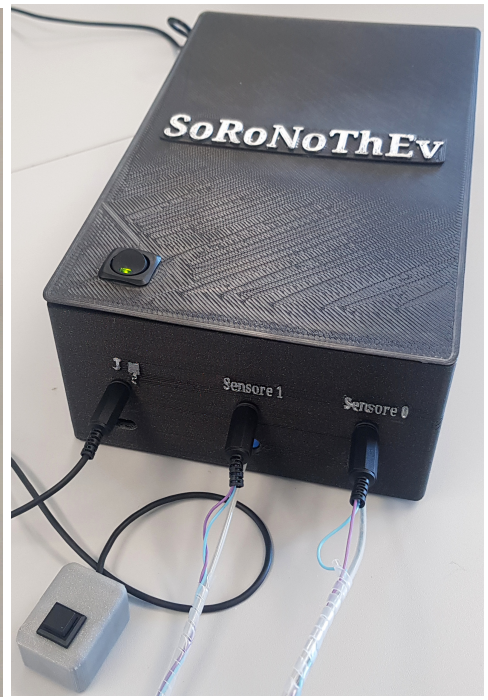

(b)

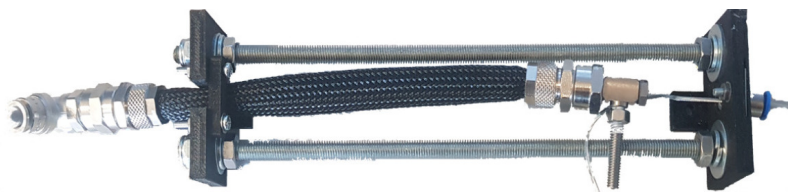

(c)

Figure 2. Main parts of the device. (c) Pneumatic Artificial Muscle (PAM).

(a) Stimulation module.

(b) Actuation and control unit.

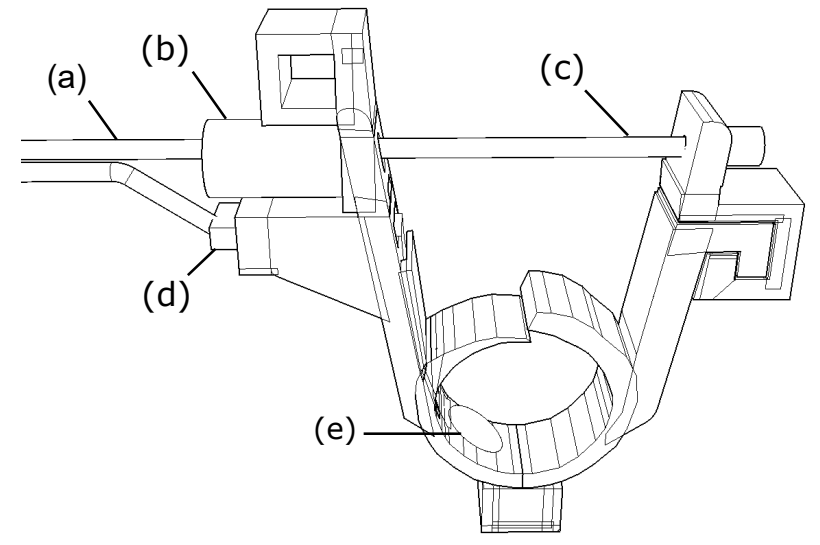

Figure 3. Stimulation ring. (a) Bowden cable. (b) Frame attach of the Bowden cable. (c) Pull rod. (d) Electric connector of the force sensor. (e) Force sensor IEE FSR149AS.

The rings are supported by cantilever beams which, in turn, are fixed to the frame in order to unload the weight of the rings on the finger articulations (see Figure 2a). Moreover, the rings are provided by interchangeable segments which can easily fit the diameter of the patient's finger. Some slider joints between each ring and cantilever beam allow to move the rings over the beam in order to reach the exact positions of the interphalangeal articulations of the fingers.

The overall structure of the device guarantees the required wearability, adaptability to the hand morphology, and, no less important, the stability of the positions of the measurement sites distributed over the articulations of the hands generally involved in the standard tests described in Section 2.

The rings are actuated by PAMs which, thanks to their natural compliance, guarantee the inherent safety during human-machine interaction and minimize the potential risk of injury. The actuation force is transmitted to the rings through a Bowden cable and a pull rod connected to the PAM (see 
Figure 3). The elasticity of the rings allows to use a single-effect pneumatic actuator and, therefore, a single PAM. Indeed, the rest position of the stimulation ring can be recovered, after the active phase of motion actuated by the PAM, thanks to the elastic return of the ring structure.

The set of PAM-actuated rings of the stimulation module, within the force control system of the device, allows the automation of the manual stimulation by the physician. As the force inputs on the patient's joints are controllable and measurable, the device solves the issue of the operator-dependent and non-repeatable evaluation of pain sensitivity thresholds.

Although the biomechatronic device for nociceptive threshold evaluation exploits the principle of the pneumatic actuation, already in use in wearable and soft haptic gloves [20], the proposed device does not overlap pneumatic gloves which are intended to generate kinesthetic feedback during the motion of the finger or tactile feedback on the finger pulp.

Compactness and lightness are the strong points of the mechanical structure of the stimulation module interacting with the patient's articulations (see Figure 2a). There are no mechanical parts placed on the anatomical sites that are not involved in nociception tests and RA diagnosis. Moreover, the Bowden cables, which transmit to the rings the actuation force from the PAMs grounded to the frame, allow to reduce, to a great extent, the overload on the patient's fingers without adverse effects to tender and swollen articulations of rheumatic patients. In a first version of the prototype, only the rings for the articulations of index and little fingers are actuated by two distinct PAMs.

Most of the parts of the device prototype are 3-d printed through the FDM 3-d printer Alfawise U20. A PLA filament Filoalfa Alfapro, which guarantees superior performance in terms of surface finishing, resistance to UV, and hydrolysis, has been used to print the case of the actuation and control unit, buttons and other components of the human-machine interface. The rings are printed by using the PLA-based filament Filoalfa Alfaplus having enhanced thermal and mechanical resistance.

The pressure source of the PAMs is provided by a $12 \mathrm{~V}$ DC roller pump, which is integrated into the actuation and control module of the device. The integrated pressure source provides an efficient and portable alternative to external air compressors.

Some piezoresistive force sensors IEE FSR149AS are installed on the finger side of each ring (see Figure 3e). The location of the force sensors is chosen in order to guarantee a stable contact between the finger skin and the sensing area of the sensor during the compression of the finger articulation. Each force signal is labeled by the name of the interphalangeal articulation of the hand. The measurement range of the miniature piezoresistive sensors is 0.1 to $10 \mathrm{~N}$ and the resistance output varies from $1 \mathrm{M} \Omega$ to $3 \mathrm{k} \Omega$. The force sensor has an active area of diameter $4 \mathrm{~mm}$; its external diameter is $8.4 \mathrm{~mm}$.

\subsection{Measurement and Digital Acquisition of Mechanical Nociceptive Thresholds}

The compression forces, which are exerted on the patient's joints, are measured through the multichannel analog/digital acquisition interface provided by the microcontroller Feather Bluefruit 32u4. The embedded control board Adafruit Feather 32u4 Bluefruit is an "all-in-one" Arduino-compatible + Bluetooth Low Energy integrated module with built-in USB and battery charging unit. At the Feather 32u4's heart there is an ATmega32u4 microcontroller running at a clock frequency of $8 \mathrm{MHz}$ (power supply 3.3 V). This board has $20 \mathrm{GPIO}$ pins, Serial and $I^{2} \mathrm{C}$ interface, SPI support, 7 PWM pins, and 10 analog inputs. The other communication module of the biomechatronic device, i.e., the Feather HUZZAH, is an ESP8266-based board (clock frequency 80 to $160 \mathrm{MHz}, 3.3 \mathrm{~V}$ logic/power) with on-board WiFi modem.

The external power supply of the device provides $12 \mathrm{~V}$ at $5 \mathrm{~A}$ max. A voltage stabilizer at $3.3 \mathrm{~V}$ is used for the microcontroller boards.

During nociceptive threshold tests, an increasing compression is applied until pain is perceived by the patient. The button in Figure 4 is pushed by the patient to report the pain threshold. Therefore, the registration of the measured value of the force threshold is triggered by the button input, before untightening the ring through the deflation of the PAM. 


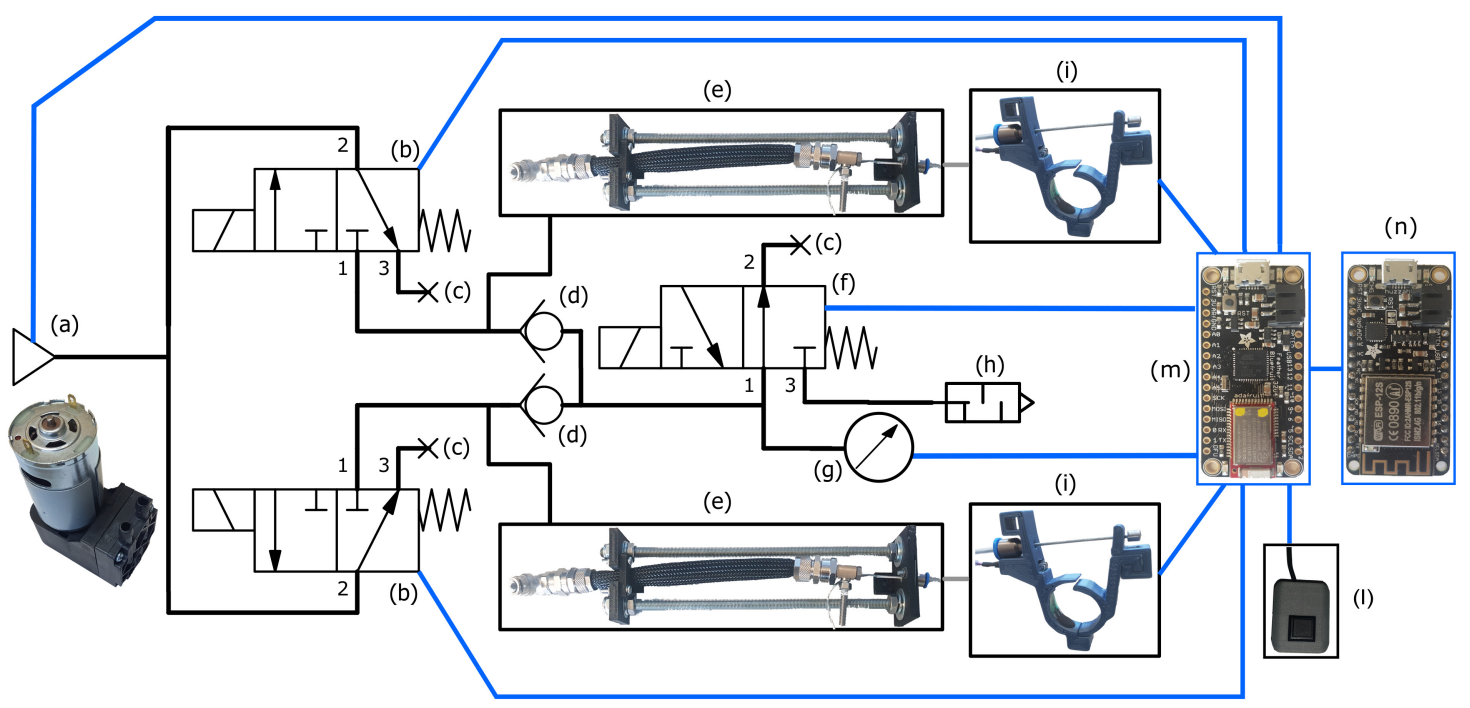

Figure 4. Schematic of the pneumatic control system. (a) Pressure Source. (b) Normally-closed servovalve. (c) Closed port. (d) One-way valve. (e) PAM. (f) Normally-open servovalve. (g) Pressure sensor. (h) Silencer. (i) Actuator ring. (1) Patient's push-button. (m) Embedded-control board Feather Bluefruit 32u4. (n) Board Feather Huzzah with ESP8266 microcontroller.

It is noteworthy that the reaction time of the patient, which corresponds to the delay between the time the subject feels the pain and the time she/he presses the button, affects the accuracy of the measurement of nociceptive thresholds. However, the current values of loading rate enable to discard the delay effect during the nociception tests. Indeed, a set point of $8 \mathrm{~N}$ is reached after approximately $3 \mathrm{~s}$ and, therefore, at the average rate of $2.67 \mathrm{~N} / \mathrm{s}$, the force variation is $0.4 \mathrm{~N}$ over a time period of $0.15 \mathrm{~s}$, corresponding to the average reaction time for humans undergoing tactile stimulation. A value of $0.4 \mathrm{~N}$ is only the $0.8 \%$ of the full-scale value of the force applied by the biomechatronic device.

Within the human-machine interface of the device, the press-button at the side of the patient is complemented by a serial monitor interface which provides, through a Bluetooth channel, both the time profiles of the compression forces applied to the finger joints and the values of the nociceptive thresholds acquired from each articulation. The visualization of force signals and nociceptive threshold values can be obtained from a smartphone screen through the execution of a BLE serial communication software.

The Wi-Fi module enables the remote transmission of pain thresholds and force signals in a cloud service which, in an extended version of the device, will include a Web interface giving a graphical output of the force profiles and measured thresholds, other than an estimate of the RA severity. The algorithm of RA diagnosis is intended to provide an index of severity of the rheumatic disease, which will be estimated by aggregating the values of pain thresholds and some dynamical properties of the force responses taken from the different articular joints of the hand through the automatic procedure of nociception measurement.

\subsection{Pneumatic Control System}

The electro-pneumatic circuit, which enables the automatic measurement of mechanical pain thresholds, is represented in Figure 4.

The current version of the prototype implements the control of the forces required for the stimulation of two different fingers/articulations. The bi-channel stimulation, which is actuated through a couple of PAMs, is obtained in a closed-loop control scheme regulating the compression force of the rings. The force signals, which are measured through the piezoresistive sensors installed on the rings, are fed back to the microcontroller Feather Bluefruit.

The pneumatic pressure in input to the circuit is obtained through a roller pump D2028B which is connected to the inlet ports of two regulation servovalves (VDW 250-6G-1-01F-Q). These valves are 
commanded by the microcontroller to regulate the air flow in input to the pressurized chambers of the two PAMs. A third servovalve (VDW 250-6G-1-01F-Q) connects to exhaust the couple of PAMs.

The pneumatic set-up, which is characterized by the presence of a common exhaust valve and two one-way valves (SMC AKH06-00), allows the reduction of the number of servovalves required to control the inflation/deflation of the PAMs. The main advantages arising from this novel and original solution are the minimization of the realization costs, reduced power comsumption, lightness/compactness, and simplicity of the device.

The pumps operates at a maximum pressure of $220.6 \mathrm{kPa}$; both the good dynamical performance and the continuous flow of the roller pump enhance the properties of controllability of the PAMs.

The force feedback control is obtained through the ON-OFF regulation of the three servovalves. Two 3/2 VDW 250-6G-1-01F-Q normally-open servovalves connect the two PAM to the pump; the third 3/2 VDW 250-6G-1-01F-Q normally-closed servovalve connect each PAM to exhaust, through two one-way valves at the inlet port of the normally-closed servovalve. The coordinated opening/closing of supply and exhaust valves enables the modulation of the air pressure in each muscle, i.e., the manipulated variable of the control scheme, thus regulating at the set-point values the intensity of the compression forces exerted by each ring.

The servovalve between the pump and the PAM, i.e., the supply valve, enables the air flow from the pressure source to the actuator chamber if the supply servovalve is switched from the normally-closed to ON (open) state, while the normally open exhaust valve turns ON (closed) in order to isolate the PAM from the environment. A set point of force to be applied to the patient's finger can be programmed on the microcontroller-based control unit. Once the force signal has reached its set point value, the exhaust valve will turn open after a predefined period of time, typically $1 \mathrm{~s}$. During the prescribed time interval, a force stimulus is kept constant on the finger, after that both (exhaust and supply) valves switch to the OFF state and, therefore, the PAM deflates in order to release the compression by the PAM-actuated ring.

A safety function of the pneumatic control system is specifically designed for the emergency condition due to the interruption of the electrical power. Indeed, the normally open configuration of the exhaust valve enables the fast deflation of the PAMs and the simultaneous release of the compression ring in the case of power outage. To improve the intrinsic safety of the device, the measurement of the PAM pressure is obtained through a pressure sensor (Honeywell SSCDANN100PGSA3); the pressure measurement is fed back to the controller to obtain some redundant information on the intensity of the nociceptive stimulation, which is very useful in the case of faulty signals from the force sensors. The fault detection enables a safe interaction between patient and device, since the compression applied to the finger can be stopped, e.g., whenever the force signal is weak while the pressure intensity is reaching a programmable safety limit.

The gauge-pressure sensor Honeywell SSCDANN100PGSA3 possesses a measurement range [0-220.63] kPa, an accuracy $\pm 0.25 \%$ of the full-scale span and an output resolution of 12 bits.

The force controller is implemented into the embedded control board Feather Bluefruit 32u4. The microcontroller sends the control signals, i.e., of activation/deactivation of the servovalves, in input to the electro-pneumatic drivers. The control signals are elaborated by the microcontroller mainly on the basis of the signals of force feedback and input on the press-button at the patient's side.

The main control parameters can be programmed on the Bluefruit microcontroller through a serial BLE interface, e.g., by setting the values of the maximum allowable compression forces, the time interval in which the compression on the patient's articulations is kept constant and the sequence of activation of the rings on the different fingers and articulations of the hands.

The signals of force, pressure and button input are sampled at $200 \mathrm{~Hz}$ and sent, via serial communication, to a second microcontroller Feather Huzzah ESP8266 through which the remote monitoring will be implemented in a cloud service.

Two are the the main control strategies implemented into the microcontroller Feather Bluefruit 32u4. The first one enables an increasing force of compression on the patient's joint. A maximum 
allowable force limit can be set in the interval of 1 to $50 \mathrm{~N}$. The control unit guarantees the quick release of the compression force by the rings whenever the press-button is pushed by the patient to report a painful level of applied force. Alternatively, the compression force can be regulated to the set point values needed to generate different levels of nociceptive input, as required for the nociception tests described in the subsequent sections.

\section{Experimental Set-Up}

The biomechatronic design and preliminary testing of the newly conceived device are the fundamental steps propaedeutic to the definition of a novel protocol for the quantitative measurement of the mechanical nociceptive thresholds in rheumatic patients. Therefore, the experimental validation of the controllability and repeatability of the nociceptive stimuli applied by the biomechatronic device to the subject's articulations has been carried out on the basis of a set of nociception tests on healthy individuals. To this end, we have exploited the main steps of the experimental procedure, originally proposed in [21] to validate a novel haptic stimulator device for the analysis of processes of human motor control and learning. In [21], the Weibull distribution is used to correlate the perceived level of the mechanical nociceptive stimulus with the intensity of the indentation applied to the subject's forearm through the haptic device.

Ten healthy subjects (aged $34.7 \pm 6.2$ years, 5 females) have been enrolled for the nociception tests. All participants have provided their voluntary informed consent. The anatomical points of mechanical stimulation, which are selected for the tests on the right hand of each subject, are the proximal interphalangeal articulations of index and little finger, respectively.

For the nociception experiments, the maximum allowable force limit is set to 50 [N] on the force controller. This limit prevents harmful and residual effects on the integrity of skin and soft tissues after nociceptive stimulation.

During the first step of the experimental procedure, each subject undergoes an increasing compression through the force exerted by the ring on the finger joint until pain is perceived. Once the patient's button is pushed, the force value is acquired. The latter identifies the maximum bearable intensity of the force stimulus, i.e., the mechanical nociceptive threshold measured through the piezoresistive force sensor installed on the stimulation ring. For each participant, the measured value of pain threshold corresponds to the reference score "level 10" within a discrete scale [0-10] of perceived pain.

An increasing sequence of five levels of compression force is afterward applied to the subject, in order to explain to the participant the subsequent phase of nociception tests. The five levels are taken as $17 \%, 33 \%, 50 \%, 67 \%$, and $83 \%$ of the maximum bearable stimulus identified as reference level. Each force stimulus is kept constant for 10 seconds.

The next step consists in a repeated nociceptive stimulation through three random sequences of 5 levels of force intensity $(17 \%, 33 \%, 50 \%, 67 \%$, and $83 \%$ of the reference level). At the end of each stimulus of $10 \mathrm{~s}$, the subject is asked to assign a score to the perceived pain on discrete scale [0-10]. A time interval of $10 \mathrm{~s}$ is left between two consecutive stimuli in order to recover the unstrained conditions of skin and soft tissues of the finger.

\section{Results and Discussion}

The profile of the pain scores $s(F)$ in response to the nociception experiments follows the Weibull cumulative distribution function

$$
s=10\left(1-e^{-\left(\frac{F-F_{\text {offset }}}{\lambda}\right)^{k}}\right),
$$

where $F$ is the compression force on the finger joint, $\lambda$ and $k$ are the model parameters to be identified, and $F_{\text {offset }}$ is the measured value of the preload which is initially regulated in order to maintain the contact between the ring and finger before starting the stimulation. 
As an outcome of the identification through the Weibull distribution, the experimental responses follow the law of variation of the perceived intensity of the pain elicited by mechanical compression (see Figure 5), already observed in the related literature (see, e.g., in [21,22]).

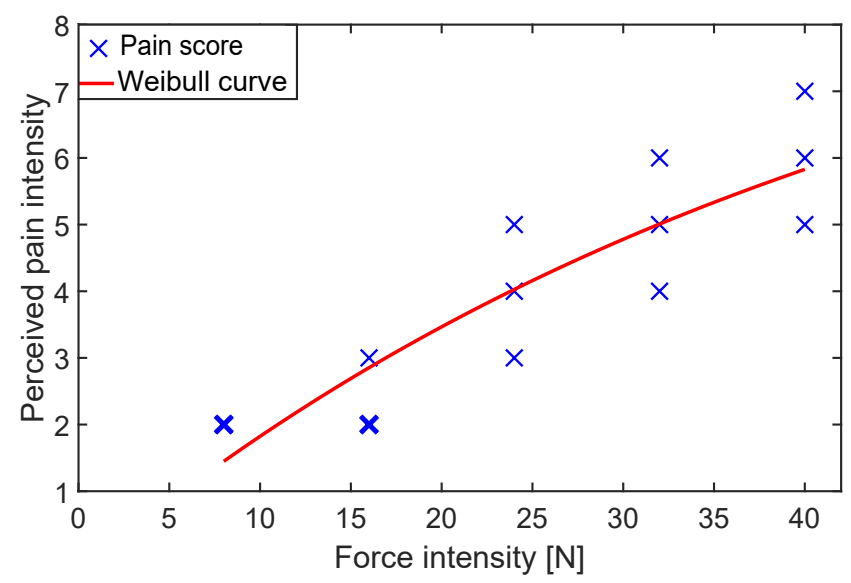

(a)

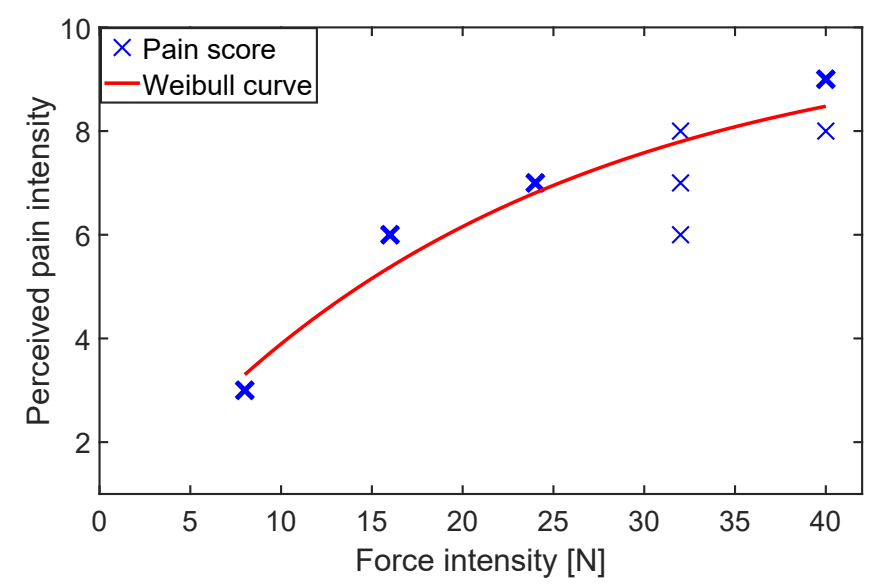

(b)

Figure 5. Typical sets of nociception scores in response to the randomized stimulation (5 levels of force $\times 3$ sequences). (a) Forefinger. (b) Small finger.

The goodness-of-fit of the Weibull model can be appreciated from the residual analysis plots in Figure 6 giving the variation intervals, the medians, the quartiles of the distributions of the residuals between the model outputs, and the data sets obtained from the Weibull fitting of the randomized tests on the forefingers and little fingers of all the participants (one Weibull curve fits 15 experimental points for each index/little finger of 10 subjects).

In all the experiments, the median of the residuals is less than 1 unit, and therefore it is possible to conclude that a similar level of pain can be evoked by the automatic stimulation whenever the same intensity of force is applied on the fingers/articulations of the specific subject.

The mismatch between the discrete nature of the nociception rating and the continuity of the Weibull function is source of approximation. However, the effects of the approximation through the cumulative Weibull distribution are mitigated since, for the standardized analysis of the residuals between the model outputs and the data sets of all experiments, the output of the Weibull distribution is discretized by sampling the function in correspondence of five levels of force in percentage of the nociceptive threshold of each subject. 


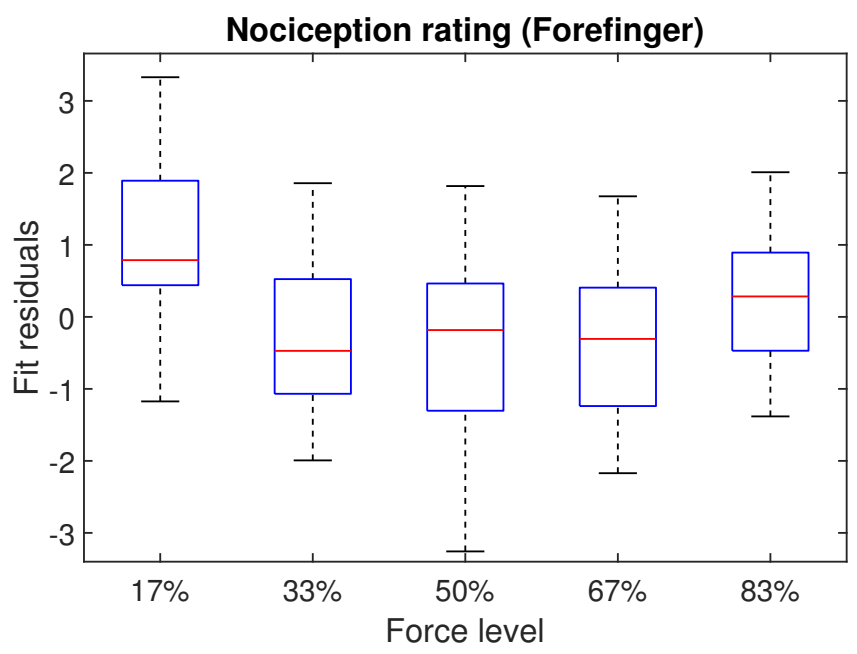

(a)

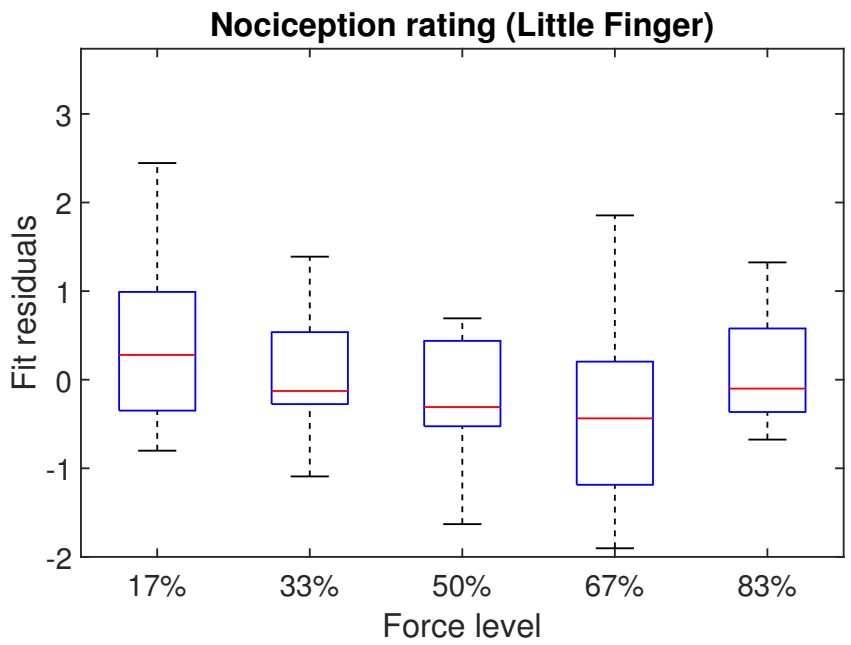

(b)

Figure 6. Box plots of the residuals of the fitting of the nociception experiments (ten fits) on (a) forefinger and (b) little finger.

The results show that the integration of pneumatic actuation with force controllers can provide satisfactory performance both in transient and steady-state conditions, also in comparison with other solutions based on series elastic mechanisms. In this respect, one of the advantages provided by pneumatic control systems is that an equilibrium position, which can achieve a set point value of force, can be maintained by closing the regulation servovalves, without further intervention by the controller or energy consumption. Conversely, series elastic mechanisms require active (and continuous) corrections by the controller to maintain an equilibrium position, in order to limit the oscillatory effects of the elastic elements on the closed-loop response.

Accurate and repeatable nociception tests have been obtained thanks to the adoption of well-characterized PAMs actuating some elastic rings applied on the patient's finger. A viable alternative to the elastic rings may be some pneumatic structures which enable to apply pressure directly on the finger joints. However, the implementation of a fully pneumatic stimulation requires the non-straightforward solution of some technological problems, due to the inherent complexity involved in the mechanical design and fabrication of the pneumatic structure, characterization of the fully pneumatic device, and, furthermore, in the design of the force controller. 


\section{Conclusions}

This works sets out with the objective of developing novel biomechatronic devices enabling the quantitative measurement of mechanical nociceptive thresholds. By exploiting all the advantages arising from soft pneumatic actuators controlled by an embedded control unit, the designed device is able to generate controllable sequences of mechanical nociceptive stimuli on the synovial joints of an individual.

As shown by the analysis of the experimental data acquired from nociception tests, the consistency of the distribution of the experimental points with the Weibull fitting of the pain perception elicited by mechanical stimuli can represent a good index of quality of the device performance, in terms of effectiveness and repeatability of the automated measurement of mechanical nociceptive thresholds.

The embedded control unit can guarantee robust and flexible performance through the execution of customized control strategies and stimulation sequences which can be implemented on the microcontroller for accomplishing nociception tests and physicophysical studies, in clinical and telemedicine frameworks.

Future works will be directed to the implementation both of a multifinger stimulation/acquisition hardware and force tracking control strategies for generating time-varying and periodic stimulations on all the joints of the hands. All the future developments can support the definition and validation of standardized and objective protocols for RA diagnosis, on the basis of the automatic evaluation of nociceptive thresholds reliably obtained through the newly conceived device.

Author Contributions: Conceptualization: L.R., R.D.G. and A.M.; Methodology: L.R., A.C., C.C., R.D.G., F.A. and A.M.; Supervision, F.A. and A.M.; Validation, L.R. and A.C.; Writing-original draft: L.R., A.C. and A.M. Writing-review and editing: L.R., A.C., C.C., F.A. and A.M. All authors have read and agreed to the published version of the manuscript.

Funding: This research received no external funding.

Conflicts of Interest: The authors declare no conflict of interest.

\section{References}

1. Cianchetti, M.; Laschi, C.; Menciassi, A.; Dario, P. Biomedical applications of soft robotics. Nat. Rev. Mater. 2018, 3, 143-153. [CrossRef]

2. Gifari, M.; Naghibi, H.; Stramigioli, S.; Abayazid, M. A Review on Recent Advances in Soft Surgical Robots for Endoscopic Applications. Int. J. Med Robot. Comput. Assist. Surg. 2019, 15, e2010.

3. Ashuri, T.; Reasnor, T.; Armani, A.; Jalilzadeh Hamidi, R.; Ahmadi, S.; Iqbal, K. Biomedical soft robots: Current status and perspective. Biomed. Eng. Lett. 2020, 10,369-385.

4. Merola, A.; Colacino, D.; Cosentino, C.; Amato, F. Model-based tracking control design, implementation of embedded digital controller and testing of a biomechatronic device for robotic rehabilitation. Mechatronics 2018, 52, 70-77. [CrossRef]

5. Andrikopoulos, G.; Nikolakopoulos, G.; Manesis, S. Design and development of an exoskeletal wrist prototype via pneumatic artificial muscles. Meccanica 2015, 50, 2709-2730. [CrossRef]

6. Capace, A.; Randazzini, L.; Cosentino, C.; Romano, M.; Merola, A.; Amato, F. Design, realization and experimental characterisation of a controllable-compliance joint of a robotic exoskeleton for assist-as-needed rehabilitation. In Proceedings of the 2020 IEEE International Symposium on Medical Measurements and Applications (MeMeA), Bari, Italy, 1 June-1 July 2020; pp. 1-6.

7. Capace, A.; Cosentino, C.; Amato, F.; Merola, A. A Multistate Friction Model for the Compensation of the Asymmetric Hysteresis in the Mechanical Response of Pneumatic Artificial Muscles. Actuators 2019, 8, 49.

8. Colacino, D.; Merola, A.; Cosentino, C.; Amato, F. Identification and modelling of the friction-induced hysteresis in pneumatic actuators for biomimetic robots. In Proceedings of the 22nd Mediterranean Conference on Control and Automation, Palermo, Italy, 16-19 June 2014; pp. 1166-1170.

9. Tanaka, Y.; Yamauchi, H.; Amemiya, K. Wearable Haptic Display for Immersive Virtual Environment. In Proceedings of the JFPS International Symposium on Fluid Power, Nara, Japan, 12-15 November 2002; pp. 309-314. 
10. Bouzit, M.; Burdea, G.; Popescu, G.; Boian, R. The Rutgers Master II-new design force-feedback glove. IEEE/ASME Trans. Mechatron. 2002, 7, 256-263. [CrossRef]

11. Tadano, K.; Akai, M.; Kadota, K.; Kawashima, K. Development of grip amplified glove using bi-articular mechanism with pneumatic artificial rubber muscle. In Proceedings of the 2010 IEEE International Conference on Robotics and Automation, Anchorage, AK, USA, 3-8 May 2010; pp. 2363-2368.

12. Du, H.; Xiong, W.; Wang, Z.; Chen, L. Design of a new type of pneumatic force feedback data glove. In Proceedings of the 2011 International Conference on Fluid Power and Mechatronics, Beijing, China, 17-20 August 2011; pp. 292-296.

13. Surendra, W.A.; Tjahyono, A.P.; Aw, K.C. Portable and wearable five-fingered hand assistive device. In Proceedings of the 2012 19th International Conference on Mechatronics and Machine Vision in Practice (M2VIP), Auckland, New Zealand , 28-30 November 2012; pp. 431-435.

14. Koo, I.; Kang, B.; Cho, K.J. Development of Hand Exoskeleton using Pneumatic Artificial Muscle Combined with Linkage. J. Korean Soc. Precis. Eng. 2013, 30, 1217-1224. [CrossRef]

15. Polygerinos, P.; Wang, Z.; Galloway, K.; Wood, R.; Walsh, C. Soft robotic glove for combined assistance and at-home rehabilitation. Robot. Auton. Syst. 2014, 73, 135-143.

16. McInnes, I.B.; Schett, G. The pathogenesis of rheumatoid arthritis. N. Engl. J. Med. 2011, 365, $2205-2219$. [CrossRef] [PubMed]

17. Shapira, Y.; Agmon-Levin, N.; Shoenfeld, Y. Geoepidemiology of autoimmune rheumatic diseases. Nat. Rev. Rheumatol. 2010, 6, 468-476. [CrossRef] [PubMed]

18. Anderson, L.; Caplan, J.; Yazdany, M.; Robbins, M.L.; Neogi, T.; Michaud, K.; Saag, K.G.; o’Dell, J.R.; Kazi, S. Rheumatoid Arthritis Disease Activity Measures: American College of Rheumatology Recommendations for Use in Clinical Practice. Arthritis Care Res. 2012, 64, 640-647. [CrossRef] [PubMed]

19. van den Bosch, J.B.; Mangnus, L.; Reijnierse, M.; Huizinga, T.J.W.; van der Helm, H.M. The diagnostic accuracy of the squeeze test to identify arthritis: A cross-sectional cohort study. Ann. Rheum. Dis. 2015, 75, 1886-1889. [CrossRef] [PubMed]

20. Pacchierotti, C.; Sinclair, S.; Solazzi, M.; Frisoli, A.; Hayward, V.; Prattichizzo, D. Wearable Haptic Systems for the Fingertip and the Hand: Taxonomy, Review, and Perspectives. IEEE Trans. Haptics 2017, 10, 580-600. [CrossRef] [PubMed]

21. Jeanneret, M.; Bagnato, C.; Allievi, A.G.; Burdet, E. A Versatile Robotic Haptic Stimulator to Study the Influence of Pain on Human Motor Control and Learning. In International Conference on Human Haptic Sensing and Touch Enabled Computer Applications; Springer: Berlin/Heidelberg, Germany, 2016; pp. 101-110.

22. Greenspan, J.D.; McGillis, S.L.B. Stimulus Features Relevant to the Perception of Sharpness and Mechanically Evoked Cutaneous Pain. Somatosens. Mot. Res. 1991, 8, 137-147. [CrossRef] [PubMed]

(C) 2020 by the authors. Licensee MDPI, Basel, Switzerland. This article is an open access article distributed under the terms and conditions of the Creative Commons Attribution (CC BY) license (http://creativecommons.org/licenses/by/4.0/). 